\title{
La Houille Blanche dans le Massif Central Français.
}

\author{
Par M. RAoul BLANGHARD.
}

(SUITE ET FIN)

L'irrégularilé des débits. - Cette irrégularité paraît d'abord un phénomène surprenant. Sauf au Sud-Est, les pluies qui alimentent les cours d'eau du Massif Central se ressentent du voisinage de la mer; elles sont fréquentes et régulières. A Limoges, le mois le plus sec (février) reçoit encore $6,6 \%$ de la pluie totale, le plus arrosé (octobre) dépasse à peine $10 \%$. Il semble que cette égalité de précipitations devrait entraîner pour les cours d'eau une véritable égalité d'humeur. Il n'en est pourtant pas ainsi. Les rivières du Massif Central sont beaucoup moins régulières quc les fougueux torrents des Alpes et des Pyrénées, et ils doivent ce grave défaut à l'imperméabilité du sol, aux conditions de pente, enfin à l'absence de l'élément régulateur que sont dans des montagnes plus élevées les neiges et les glaciers.

Il n'est pas besoin d'insister sur l'imperméabilité. Toute la carcasse du Massif Central, faite de gneiss et de schistes, de granite et granulite, est imperméable. Il n'y a de perméable, à des degrés divers, que les masses volcaniques d'Auvergne, du Velay et les plateaux calcaires des Causses. Les bassins eux-mêmes ont un sol imperméable d'argile ou de marno-calcaires. Ainsi la superficie des terrains à travers lesquels l'eau peut filtrer n'atteint pas un septième de l'ensemble. Les pluies qui tombent sur ce socle imperméable vont donc aussitôt rejoindre les cours d'eau qui enregistrent dès lors fidèlement toutes les pulsations de la pluviosité. N'insistons pas trop cependant sur cette condition d'irrégularité. Il existe dans les parties élevées de l'Oựest et du Centre nombre de prés humides, marécages, tourbières, qui font l'office de réservoirs et régularisent les apports de la pluie ou la fusion de la neige. N'oublions pas non plus qu'en montagne, lorsque la pente est forte, il n'y a pas de perméabilité qui tienne, et que le ruisellement s'effectue aussi bien sur les roches perméables que sur les autres.

La pente est donc un coefficient plus actif d'irrégularité. Il semble qu'après avoir longuement démontré et déploré la faible déchivité des talwegs, nous puissions recueillir maintenant le bénéfice de cette disposition et estimer que l'écoulement des eaux se fait avec lenteur, au profit de la régularité du régime. Hélas! Ce qui est une faible pente pour l'ingénieur soucieux d'alimenter ses turbines est une terrible déclivité pour l'eau courant à la mer. Pour un hydrologue, 2 à 3 mètres par kilomètre représentent une pente torrentielle, et à ce compte-là tous les cours d'eau du Massif Central sont des torrents. Pour s'en çonvaincre, il n'est besoin que de constater la vitesse de translation des crues. Ne parlons pas de l'Ardèche et de ses voisines, dégringolant du Sud-Est du massif vers le Rhône ou la Méditerranée, et où les crues se propagent à une vitesse de 12 à 15 kilomètres à l'heure, et parfois $5 \mathrm{~m}$. 50 par seconde ; il s'agit ici de rivières où la masse formidable des eaux à déverser s'ajoute à la raideur des pentes pour accroître la vitesse d'écoulement. Mais sur la Loire en amont du Forez, le maximum d'une forte crue se propage à 10-12 kilomètres à l'heure et il ne lui faut pas plus de 3 jours pour franchir les 354 kilomètres qui s'allongent du Velay au bec d'Allier. La vitesse est plus forte encore sur l'Allier où, entre Langogne et le confluent avec la Loire, les $353 \mathrm{ki}$ lomètres ont été parfois dévorés en 48 heures $(7 \mathrm{~km} .4$ à l'heure, bien que le trajet comporte une grande étendue de plaines). Sur le haut Tarn, entre Florac et Millau $(85 \mathrm{~km}$.), la vitesse est de 15 à 16 à l'heure. Il en est de même sur les rivières de l'Ouest dont la pente est aussi forte. Dans ces conditions, on comprend que ces pentes, susceptibles d'évacuer en quelques heures, ou au moins en quelques jours des crues énormes et de laisser ensuite tomber rapidement le débit, puissent être considérées comme un élément efficace d'irrégularité. Pourtant, n'exagérons rien. Dans les Alpes, l'Isère naissante, dans le val de Tignes, affectée d'une pente formidable dépassant 60 mètres au lilomètre, est un des cours d'eau les plus réguliers qui soient ; le rapport entre maximum et minimum absolus en sept ans d'observations est inférieur à 14 . La pente aggrave, souligne l'irrégularité ; elle n'en est pas la cause. Ce qui manque ici, c'est une quantité suffisante de neige et de glaces.

Les Arvernes vont se récrier, et assurer que les hivers normaux voient tomber sur leurs montagnes une couche épaisse de neige qui dure, dans les coins bien abrités, jusqu'à la fin du printemps. Personne n'y contredira, et nous pensons même que certaines hautes régions en sont mieux pourvues, à altitude égale, qu'une grande partie des Alpes du Sud. Sur les sommets du Velay, elle tient généralement de décembre à avril. Dans la " montagne " du Limousin, elle peut persister deux à quatre mois sur le sol. Les pentes supérieures du Cantal restent longtemps blanches, surtout sur le versant septentrional. Mais ces hautes terres ne sont que des taches éparpillées à travers le massif, et, sur la plus grande partie de son étendue, la 'neige n'est guère qu'un accident, toujours éphémère ; un mànteau sans épaisseur, dont la fusion, vite achevée, n'a jamais qu'une influence secondaire sur le régime des rivières et ne se produit en tous cas qu'à contre-temps, à une saison où le débit est encore abondant par ailleurs. Quant aux glaciers, il ne peut plus en être question. Les accumulations de neige tassée par le vent, les congères, ne peuvent, en dépit de la lenteur de leur fusion, rappeler même de loin, le rôle des glaciers dans l'alimentation des cours d'eau.

Peu de neige et pas de glaces, en dépit de l'humidité du climat, voici qui doit encore être mis au compte de l'altitude. Et les conséquences n'en sont pas minces. Dans les Alpes, dans les Pyrénées, si on laisse de côté les rares régions où la neige est un phẻnomène exceptionnel, la saison chaude est, grâce aux neiges et aux glaciers, l'époque des hautes eaux. Même dans les petits massifs extérieurs de faible altitude, les Préalpes, la baisse des cours d'eau à la fin de l'été est rarement aussi accentuée qu'en hiver. C'est là un immense service rendu aux rivières et qui leur assure une véritable régularité. On se rend aisément compte que l'été, saison de chaleur, de soleil, d'évaporation, est pour les cours d'eau de nos climats la période critique, dangereuse, où les guette la pénurie ; car en hiver, l'évaporation et l'infiltration étant réduites au minimum, la moindre pluie, le moindre apport profiteront aux rivières, et même en montagne il se trouve toujours un coup de vent chaud, une poussée de föhn, pour faire fondre les neiges les plus basses et garnir ainsi les talwegs. Mais le Massif Central est presque entièrement privé de cette bienfaisante compensation qu'apporte en été la fusion des neiges, et le voilà livré tout uniment à la seule influence de la répartition saisonnière des pluies.

$\mathrm{Ce}$ n'est pas que cette répartition soit défavorable. Au contraire, elle est aussi favorable que possible, puisqu'elle fait tom- 
ber le maxmmun de pluie a l'épocpue nu les rivières ont le plus besoin de renfort, et le minimum a la saison oì elles se defendent le mieux. Cest en ele et au debul de lautomme que tombent les quantilés les plus fortes, sur loule l'elenduc du massil. Sur le haut Cher, inin, juillet, octobre viement en tète des autres mois: sur la Creuse, oclohre, juin, juillet, puis aont. J)ans les bassins de la looire supérieure, de l'Allier, juin, mai, juillet sont les mois de maximum ; viennent ensuile or lobre, puis septembre et aont. Octobre, juin, mai sont en tète sur la haute Vienne, sur la haute Dordogne. dans les bassius superieurs du fol el du Tarn. A part le versant Sud-Est, déjà méditerranéen, il est donc acquis que dans le Massif Central les pluies. d'ailleurs assez. également réparties, tombent en plus grande abondance à l'époque où les rivières en ont le plus besoin. Célébrons cette heureuse concordance! Mais les rivières paraissent bien peu en profiter. Car il est remarquable que tous les cours deau du Massif Central ont leur maximum en hiver, lorsqu'il pleut le moins, et leur minimum en été, lorsqu'il tombe le plus d'eau. Qu'il y ait ainsi décalage, cela n'a pas, en soi, grande importance. Mais le phénomène indique clairement que les pluies, privées du renfort des neiges, ne sont pas capables à la saison critique d'assurer la régularité du débit. Ce défaut de concordance entre l'alimentation pluviale et le débit des cours d'eau est un premier témoignage d'irrégularité. Mais il y en a d'autres, plus graves : la différence entre les débits moỳens, la différence entre les débits extrêmes.

La démonstration est vite faite que le régime moyen de ces rivières comporte des hautes eaux d'hiver et des basses eaux d'été. Février, qui est avec janvier dans tout le massif le mois où les précipitations sont le plus faibles, est aussi celui oủ l'on trouve les débits moyens les plus forts sur la Loire à Digoin, l'Allier à Moulins (à égalité avec avril), le Cher à Montlucon, la Creuse supérieure, la Vienne, la Vézère. Mars tient le même rôle sur la Dordogne à Souillac, sur la Cère inférieure et vraisemblablement sur la Truyère, le Lot. En revanche, août et septembre comportent partout le minimum le plus accusé. C'est exactement le contraire de ce qui se passe dans les Alpes, où les basses eaux sont en hiver et les hautes eaux en été, et cette opposition n'est d'ailleurs pas un mal pour l'industrie hydroélectrique, puisqu'on peut entrevoir une possibilité de compensation des deux régimes l'un par l'autre. Notons cependant que dans les parties les plus élevées, sur la haule Dordogne, la Cère supérieure, la haute Truyère, qui sont de vraies rivières de montagne, le régime est plus complexe et comporte outre l'étiage d'été un minimum secondaire d'hiver, cerné par deux maxima de printemps (mars, avril) et d'automne (novembre, décembre), ce qui rend l'utilisation plus complicjuée encore.

D'autre part, entre ces débits d'hiver et d'été, la disproportion est grande. Considérons les volumes moyens mensuels qui, sur ces cours d'eau capricieux, ne sont que des abstractions jamais réalisées. Nous voyons que sur le Cherà Montluçon, pour treize ans d'observations (1888-1900), la moyenne du mois le mieux fourni (février) est 15,2 fois plus élevée que celle du mois le plus maigre (aônt), rapport énorme. Sur la L.oire supérieure, sur l'Allier, les contrastes sont de mème ordre. Sur la Dordogne à Souillac, les moyennes obtenues à la suite de l'étude de huit années pluvieuses donnent encore au débit de mars une puissance 4,1 fois supérieure à celle du débit de septembre, et c'est là une des rivières les plus constantes, les mieux alimentées du massif. Regardons les Alpes : dans les Préalpes du Nord, le rapport entre mois extrêmes est partout inférieur à 4 : oil tombe à 2,4 sur le Guiers-Vif ; dans les Préalpes du Sud-Ouest, les plus sèches, ce rapport sur la haute Drôme ne s'élève qu'à 11,3 .

Mais ceci n'est rien à côté des écarts entre extrêmés. C'est là que se traduit le mieux l'énorme différence entre le régime des cours d'eat alpuns of celui du Massif Central. C'esh enrore la Dordogne a Souillac qui se manileste la plus régulière ; le rapport entre les extrèmes observés pendant huit ans est comme 1 pẩ rapporl à 83. En un an, sur la l)iège, les chiflres de débil oul varié de 1 à 150, el sur le Taurion de 1 à 200 . P'our la Cère, a Laroquebrou, le rapport pour une dianine damnes d'observations est également voisin de 200. On l'évaluc, le lacon asse\% arbitraire, à 220 pour la Vienne ; pour le 'Tarn. il faul l'élever à 266. El ce sopt là les chiffres modérés, ceux des rivieres paisibles et bien garnies. Avec la Creuse a Argentun, c'est 148 : lil Loire à Digoin, 520 ; l'Allier supérieur, 589 ; la 'Truyère, 600. Sur de petites rivières de montagne, a forte pente, les chiffres atteignent el dépassent $1.000,2.000$. I a palme cependant semble jusqu'ici (car n'oublions pas que nous sommes asse\% mal inlormés) revenir au Cher supérieur. Celui-ci a vu, dans l'élé 1906, son débit tomber à 30 litres par seconde au droit du barrage de Rochebut : dix mois avant, le 9 novembre 190.), lariviere roulait au mème endroit plus de 100 mètres cubes. Le rapport entre extrêmes est donc ici supérieur à 20.000 .

Combien modérés, à côté de ces fantaisies. paraissent les écarls de nos torrents alpestres! En huit ans d'observations sur la haute Isère à 'Tignes, le rapport entre extrèmes n'est que de 1 a 14, et pourtant nous sommes en pleine montagne. Sur la basse Isère à La Sône (14 ans d'observations), ce rapport ne dépassé pas encore 14,4 . Il est de 19,5 pour la Durance à Briançon, de 21,7 pour l'Isère à Mottiers, de 53,5 pour la Durance à Mirabeau (14 ans), de 72 pour l'Ubaye à Barcelonnette. Il ne s'élève audessus de 100 que dans les Préalpes, où les neiges disparaissent plus vite, et pour trouver des chiffres comparables à ceux du Massif Central, il faut aller jusqu'aux rivières méditerranéemnes : le Buëch (581), l'Eygues supérieure (3.200), la Dròme. Contentons-nous de comparer une des rivières les plus favorisées du Massif Central, la Cère à Laroquebrou (800 kilomètres carrés de bassin) avec un cours d'eau équivalent des Alpes, l'Isère en amonl du Doron de Bozel (bassin de $907 \mathrm{~km}$. carrés). Les observations concernant la Cère portent sur 10 ans (1909-1918), celles de l'Isère sur 13 ans (1903-1915). Les maxima absolıs diffèrent peu : 220 niètres cubes pour la Cère, 202 pour l'Isère. Mais l'Isère n'est jamais descendue à 9 mètres cubes, la cère est tombée à moins de 2. Le débit caractéristique d'étiage, c'est-à-dire celui au-dessous ducuel la rivière n'est pas descendue plus de 10 jours par an, est de 11,6 mètres cubes à Mo:tiers. de 2,6 à Laroquebrou, correspondant à des apports par kilomètre carré de 12,7 liLres en Savoie, de 3,2 litres en Cantal. On voit i quelle indigence, si l'on compare aux conditions alpestres, est réduite en cté une rivière dont le bassin reçoit pourtant les quantites les plus considérables de neige de tout le Massif Central. l,irrégularité de débit jusque sur les cours d'eau les moins impropres à l'ulilisation. est flagrante.

Voilà le bilan des conditions physiques. II n'est pas trìs engageant. Des pentes insulfisantes, qui permettent marement l'aminagerer unc chute, même basse, à peu de frais. Un débil médiocr, et c'est aulant de perdu pour la puissance des installations, d'autant qu'on ne dispose que de faibles hauteurs de chute. Un débit irrégulier, et c'est grave, car avec les laibles dénivellations des cours d'eau, il n'est pas possible, dans ces conditions, de se procurer nue grande quantité de kilowalls permanents. Iićpétons-le : par comparaison aver les Alpes el les Pyrénées, l'inferiorité est manileste.

Les hommes n'ont eu que plus de mérite à s'en accommoder. 


\section{II. - L'ÊVOLUTION DE LA TECHNIQUE}

Les premières réalisations effectuées dans le Massif Central ont été de type alpin ou pyrénéen. Il ne pouvait en être autrement. On avait lä de l'eau qui coulait, rapide et inutile ; conment ne pas essayer de l'emplover suivant les formules qui avaient réussi, avec tant d'éclat, dans les Alpes? On chercha donc à tirer parti de ce qui ressemblait le plus aux chutes alpines, à ces dénivellations de 150 à 250 mètres qui avaient lancé la houille blanche. Il ne fallait pas être trop difficile et espérer trouver seulement de moitié aussi bien. Mais on pouvait essayer d'exploiter, avec aussi peu d'aménagements que possible, el presque à l'état brut, les dénivellations les plus raides réalisées a et là au hasard des progrès du creusement, à condition que le débil lût sulfisant et ne descendìt jamais all-dessous d'un minimum utilisable. Comme dans les chutes alpines de ce genre, l'équipement devait être aussi réduit que possible ; un barrage léger, donf l'unique role élait de diriger vers la dérivation la quantité d'ean nécessaire; une dérivation de faible longueur ; enfin la conduite forcée aboutissant aux turbines, et qui représentait la plus grosse partie de la dépénse. Au fond, il s'agissait de tirer parti des cas exceptionnels, on pourrait presque dire tératologiques, par lesquels les cours d'eau du Massif Central offraient quelque ressemblance avec ceux des Alpes.

Voilà ce qu'on a presque uniquement conçu el réalisé avant la guerre. Le type le plus parfait est peut-être l'installation du Saut-du-Tarn. Passant des roches cristallines du Massif Central à des dépôts teriaires faits d'argiles peı résistantes, le Tarn dégringole brusquement, sur 150 mètres de longueur, d'une hauteur de 10 mètres : les eaux, comme celles de la Valserine au pont des Oules, se répandent en une foule de chenaux tortueux qui griffent la pierre, aboutissent à des conduits moins nombreux, plus profonds, anastomosés entin en un chenal terminal où l'eau se précipite en bouillonnant avant de redevenir une calme rivière. Voici longtemps, au début du $\mathrm{XIX}^{\mathrm{e}}$ siècle, un barrage très sumple permit de dériver vers chaque rive une partie des eaux de la rivière, pour les faire basculer, 100 mètres plus loin, droit sur les 10 mètres de chute ; aujourd'hui, la dérivation de gauche (Saint-Juéry) donne une puissance normale disponible de 1.300 kilowatts (1), celle de droite (Arthez) 740 , soit à elles deux plus de $2.000 \mathrm{kw}$. A quatre kilomètres en amont, la pente, déjà forte, donne 515 kilowatts aux Avalats ; le tout est utilisé dans la puissanté usine des Hauts-Fourneaux, Forges et Aciéries du Saut-du-Tarn, dominant les rapides de la masse puissante de ses bâtiments étagés, d'où une douzaine de hautes cheminées menacent le ciel (pl. I).

Il existe d'autres cas presque aussi démonstratifs. Sur la Vézère, nous savons que la rivière, en aval d'Uzerche, étreinte dans une gorge de quartzites et de schistes résistants, y roule avec rapidité et culbute mème au Saut-du-Saumon dans une petite chute. On n'a pas manqué de profiter de ces heureuses dispositions. Un canal en souterrain, coupant une boucle de la vallée, est allé recueillir à un kilomètre en amont de l'usine un débit de 6 à 8 mẹtres cubes ; il gagne ainsi une chute brute de $44 \mathrm{~m} .50$ et permet de produire dans l'usine du Saillant une puissance moyenne de 2.250 kilowatts, qu'utilise la Compagnie d'Electricité de Limogés. A l'amont immédait, sous Pouch, on a barré la rivière de façon à utiliser encore un débit maximum de 30 mètres cubes sous une chute brute de 2 mètres, ce qui donne me. puissance moyenne de 250 kilowatts. Sur la Vienne, la rivière

(1) Conformément aux instructions du Service des Grandes Forces Hydrauliques, nous estimerons les forces en kilowatts de puissance normale disponible. Celle-ci est le produit de la hauteur nette moyenne en mètres par le débit moyen annuel en mètres cubes devient torrentueuse en aval d'Eymontiers ; on y' a installé, en profitant également d'un méandre, l'usine de Bussy-Varache, qui donne 1.750 kilowats aux Tramways de la Haule-Vienne, tandis qu'en aval les Papeteries du Iimousin recreillent 200 kilowatts à Farebout. Dans les parties à profil raile du talweg de la Creuse, on a logé dans les prenières années du $x x^{e}$ sic̀cle, les petites usines de Chantegrelle (Houillères d'Ahun), Bonnavaud (Société Electrique de (iuéret), Roche-Fat-l'Aigue (Centre Electrique), de 500,400 et 400 kilowatts de puissance normale. La Dore, à l'issue du bassin d'Ambert où sa pente est extrêmement réduite, s'encaisse dans les granites du I ivradois avant de rejoindre la Limagne, et sa pente y varie de 5 à 10 mètres au kilomètre. Aussitôt, voilà deux usines, celle d'Olliergues, à la Société des Papeteries d'Auvergne, avec 350 kilowatts ; celle de Sauviat qui fournit 1.400 kilnwatts à la Société des Forces Motrices d'Auvergne. Enfín la Yis, afluent de l'Hérault, presque seule sur ce versant Sud-Est, est utilisable parce qu'elle naît rans les Causses de grosses sources qui soutiennent le débit même l'été; de ce côté, la pente ne manque pas ; une dérivation, d'ailleurs assez longue, amène un débit moyen de 2,6 mètres cubes sur une chute de 106 mètres, la plus haute qui ait été réalisée dans le Massif Central au cours de cette période ; mais ce rebord Sud-Est est si peu le Massif Central : La puissance normale, utilisée par le Sud-Electrique, cst de 1.755 kilowatts. Ajoutons-y la chute de Truscas sur l'Orb supérieur, 49 mètres, donnant 520 kilowatts à la Société biterroise de Force et Lumière.

Dans une partie distincte du Massif Central, il y avait aussi des raisons pressantes de se procurer de l'énergie. Le bassin supérjeur de la Loire jusqu'à Roanne, voisin des groupes industriels de Saint-Etienne et Lyon, a une faim aiguë de kilowatts pour ses tissages, sa métallurgie, sa papeterie. Il en demande bien aux Alpes, mais il tient à s'en procurer sur place la plus grande quantité possible. Les rivières sont mal alimentées ; les irrégularités du régime $\mathrm{y}$ sont formidables. Mais il y a de la pente, et surtout on a besoin de courant. Voilà 25 ans que des usines hydro-électriques s'y sont installées, en dépit de conditions physiques médiocres. Le fleuve, où la pente est déjà ralentie, n'anime que de faibles installations : 1.000 kilowatts en deux usines en amont du Puy (Energie électrique des Cévennes), 500 à Saint-Victor, 700 à la Vourdiat, enfin 200 pour les papeteries de Villerest. Les affluents, plus rapides, plus sauvagement encaissés, fournissent beaucoup plus : sur le Lignon vellave, 1.700 kilowatts, dont 1.500 à Château-de-Lignon; sur l'Ance du Nord, la grosse usine d'Ance, 5.000 kilowatts ; sur le Lignon forézien 1.200, dans les usines de Saint-Martin et Vaux. Ainsi, dès avant la guerre, on disposait là d'une force de 10.400 kilowatts c'est-à-dire presque autant que dans tout le reste du massif, et cela dans une des régions qui se prêtent le moins bien à cette captaion de l'énergie. De cette constatation on pouvait du moins tirer un enseignement : c'est que le besoin de lilowatts serait capable de faire passer par dessus les difficultés techniques des entreprises.

Mais jusque dans cette région privilégiée, on s'était contenté de chercher quelques emplacements favorables, sans vouloir se lancer dans de grands travaux que l'irrégularité et la faiblesse du débit semblaient rendre trop coûteux pour qu'ils pussent payer. Donc, on n'avait guère fait que glaner les possibilités exceptionnelles, et le moment approchait où l'on en aurait épuisé la liste. En continuant l'emploi de cette technique, les progrès de l'aménagement n'allaient pas tarder à être arrêtés. D'autre part, on se condamnait à ne disposer que de petites installations : les pentes de ces rivières, mème lorsqu'elles passent pour fortes, ne sont pas suffisantes pour donner de hautes chutes, et les débits, non corrigés, fournissent trop peu. Les plus grosses de 
ces usmes, à l'exception de celle de l'Ance, dépassaient à peine 2.000 kilowatts de puissance normale. Donc peu de chutes, et d'un faible rendement. En ajoutant à celles que nous avons énumérées 400 kilowatts produits sur la haute Dordogne, 700 sur la Diège et la Triouzoune, ses affluents de droite, 300 que la Société de la Vieille Montagne tirait du Lot à Capdenac, 550 empruntés à I Alagnon (Lempdes et à la Couze de Chambon (les Granges). on arrivait pour tout le Massif Central à une puissance moyenne normale d'à peine 25.000 kilowatts. Ce n'est guère plus que ce que donnera à elle seule la grosse usine alpine de Véclaire, installée suivant les mêmes principes, mais disposant du débit d'un énorme torrent dont la pente est brusquement exaspérée par la traversée d'un magnifique verrou glaciaire.

La récolte était mince, mais les besoins étaient énormes. Les petits bassins houillers de l'Ouest, thun, StEloy, Brassac, Aubin, Carmaux, ont déjà de grosses industries à satisfaire ; le moindre accroissement industriel les laisserait essouflés, insuffisants. D'autre part, à proximité, il y a d'immenses régions à desservir : tout le bassin de la Loire, celui de la Garonne, le pays de la Charente, où l'unique source d'énergie est le charbon acheté à grands frais aux Anglais el transporté à frais non moins considérables vers l'intérieur. Pour tout ce qui s'étend au Nord de la Garonne, le Massif Central peut s'assurer là, en fourniture d'énergie, une véritable chasse gardée. Enfin, il y a les chemins de fer à électrifier ! Tant de besoins lorsque l'Allier, le Cher, la Creuse, la Vienne, la Vézère et leurs affluents étaient à peine utilisés, et qu'on n'osait pas aborder les plus puissantes rivières du massif : Dordogne, Rhue, Cère, Truvère, dont les eaux grondant dans des gorges solitaires roulaient, inutiles, hostiles, vers la mer !

Il était fatal que naquît et prît corps l'idée d'une technique nouvelle, qui permît de tirer parti de ces ressources inexploitées. Au lieu de subir passivement les imperfections et les caprices de ces cours d'eau, il fallait s'appliquer à les corriger. D'immenses possibilitès deviendraient dès lors disponibles. Or les défauts de ces rivières étaient bien connus : insuffisance de la pente, pénurie d'alimentation et surtout irrégularité du débit. S'il est difficile de remédier à la pénurie d'alimentation parce qu'il n'est pas encore au pouvoir des hommes de faire pleuvoir ou neiger à volonté, du moins les effets de ce défaut, qui sont graves lorsqu'on utilise les chutes à l'état brut, s'atténuent et disparaissent si l'on peut relever leur hauteur et régulariser le débit. Un mètre cube tombant de 10 mètres vous donnera 100 chevaux; hissez-le à 100 mètres, il vous en donnera 1.000 . La modicité du débit n'a dès lors plus d'importance; si, en outre, vous obtenez qu'au lieu d'avoir tantôt 500 litres et tantôt 2.000, vous puissiez amener régulièrement 1 mètre cube sur vos turbines, tout sera pour le mieux. C'est donc au défaut de pente et à l'ir- régularité de régime qu'il fallait s'en prendre. Or ils comportent fort heureusement un remède commun, le barrage.

Il ne s'agit pas assurément de ces minces obstacles comme on ell a disposé en travers des rivières alpines ou pyrénéennes pour guider les eaux vers une chambre de décantation à l'origine de la dérivation. Ce qu'il laut ici, ce sont d'énormes ouvrages barrant, presque de bas en haut, toute une vallée et transfornuant l'amont en lac ; en un mot, c'est le barrage-réservoir, comme on songe fort à en installer en quelques endroits bien choisis des Alpes : Génissiat sur le Rhòne, Chambon sur la haute Romanche, Serre-Ponçon sur la Durance, Caréjuan sur le Verdon, Derrière le barrage, haut de 40,50,60 mètres et plus s'il le faul, on emmagasine des millions de mètres cubes, el voici le débit régularisé: si l'on y met le prix et si le réservoir est suffisamment vaste pour pouvoir emmagasiner la quantilé que représente le coefficien! d'irrégularité, le débil pourra être constant. D’autre part, l'eau évacuée du haul du barrage représente une chute dont laménagement n'ast yu'un jeu : les conduiles forcées qui jaillissent du- halrage descendent droit sur lusine installée à la base do l'ouvrage. L'on évite ainsi unc dérivation de cinq, dix lomètres amenamt un débit restreint et irrigulier.

Cette solution a ses inconvénients. Elle coûte cher, très che"

Elle amène la submersion totale de la vallée en amont, sur une distance souvent considérable, de sorte qu'il faut compter non seulement avec le prix de la construction du barrage, mais avec des indemnités d'expropriation, des déplacements de routes, de ponts, de villages même. Il faut prévoir le risque d'envasement, si redoutable sur les rivières torrentielles ; le petit barrage d'Avignonet, sur le Drac, a vu son réservoir envasé en quinze ans. D'autre part, quelle que soit la solidité de l'ouvrage, sur lui plane, même voilée, la menace d'une rupture et du cataclysme qui s'ensuivrait. Voilà de quoi faire réfléchir. Mais on ne pouvail nier, en revanche, que les vallées du Massil Central semblassent pour la plupart admirablement disposées pour le succès de ce type d'aménagement. Ces vallées sont élroites et leurs parois raides : ce sont souvent de vraies rainures enfoncées de cent à deux cents mètres dans la masse des plateaux. I.e barrage représente donc un cube assez restreint de matériaux, el la roche encaissante est dure, solide. Ces gorges profondes sont peu fréquentées par l'homme. On n'y trouve guère de roules, guère de ponts, peu ou pas de champs; Ia place manque, el les débouchés. L'habitation humaine y est unc rareté : çà et là, de pauvres hameaux sur un replat et, au fond, quelques moulius, qui se défendent d'ailleurs contre l'expropriation avec une belle opiniâtrelé d'Arvernes ou de Cadurques. I a submersion de ces gouffres à peine fréquentés n'entraîne donc ni grands dommages, ni grands frais. Puis, la pente est faible ; le barrage n'a donc pas 
besom d'être très haut pour emmagasiner dans le réservoir un énorme volume d'eatr. Enfin, la pente étant peu déclive et la roche généralement dure, ces rivières roulent peu d'alluvions. Les sables de la Loire el de l'Allier ne semblent venir en abondance que des bassins du Forez, de Limagne et du Bourbonnais. L'envasement des réservoirs ne paraît donc pas une éventualité monaçanle ; ceux qui ont été aménagés déjà en témoignent.

Concluons donc que, s'il y a à propos de cetle technique du pour el du contre, pen de régious se prêtent aussi bien que les vallées du Massil Central à l'installation de barrages-réservoirs. On y trouvera d'ailleurs de gros avantages. La technique du début n'elail qu'une sorte de gaspillage, tirant localement de condiijons extrememenl lavorables un très médiocre parti ; tandis qu'un barrage régularise le lébit d'aval et peut faire sentir son influence sur plusieurs installaLions successives. Enlin on n'a pas le choix. Si lon veut meltre en valour les énormes forces que recelent les rivieres du massif, e'est par la cu'il faut en passer.

Il y a déjp̀ quelque lemps qu'on s'en est aperęu. On n’avait d'ailleurs qu'à s'inspirer dexemples fournis par le pays même. Un pen partoul on y employail des relemues pour acliommer des moulins. Dans la Combrailles, ou bassin du haut Cher, depuis des sic̀les on remérliait à l'irrégularilé el à la pénurie des ruis-

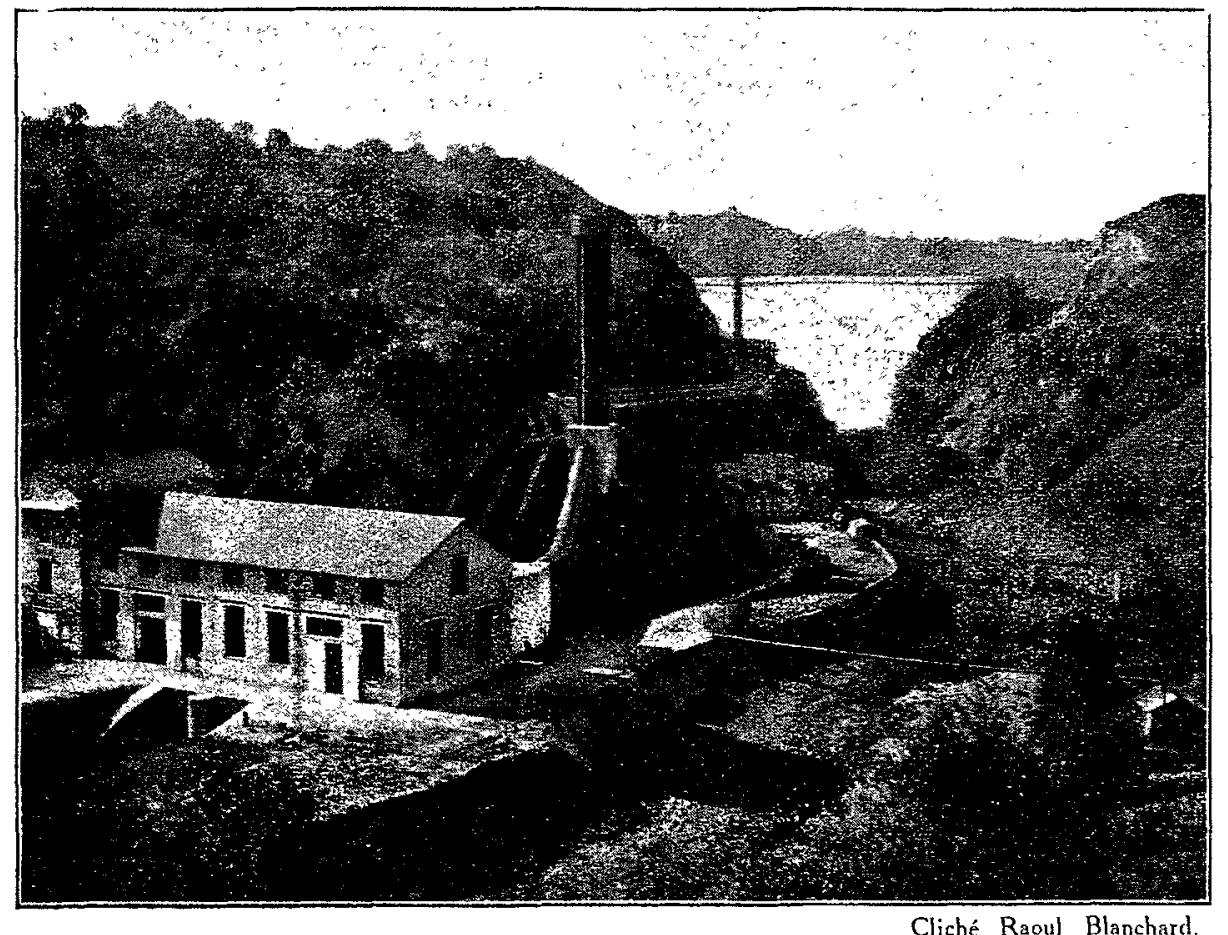

Pl. I-B. - Barrage el usine de Rochebut, sur le Cher. lowatts. Qu'on juge de ce qu'il aurait fallu de longueur de dérivation, avec le débit inconstant de la rivière, pour obtenir seulement la moitié de cetle puissance ! Car pour l'obtenir tout entière, il y avait une totale impossibilité.

C'est en 1904 que fut mise en service l'usine de Queuille. Dès ce moment, on se préoccupait d'imiter cet exemplesur le Cher. A une dizaine de kilomètres du point où la rivière, définitivement formée par la convergence de la Tardes, de la Voueize et du haut Cher, atteint le bassin de Montluçon, Ja vallée s'étrangle entre des parois très raides mordant sur une belle roche dure (une granulite à grain fin), tandis qu'en amont elle est un peu moins à l'étroit dans des gneiss. La, pente est déjà laible puisque dans cette section, entre les kilomètres 12 et 23 comptés à partir de Montluçon elle ne comporte qu'une déclivité de $6 \mathrm{~m}$. 41 au kilomètre. Donc, des conditions physiques favorables à l'établissement d'un barrage el d'un réservoir; d'autre part, un très grand besoin de cet aménagement, le Cher étant, comme on l'a vu, abominablement irrégulier et l'industrie de Vontluçon réclamant ardemment de l'énergie. Aussi a-t-on installe sous le hamean de Rochebul (commune de TeilletArgenly) un barrage do 43 mèlres de haul, derrière lequel un lac s'allonge dans le's gorges, remontant le Cher sur 2.500 mètres, puis bifurquant dans la Tar- seaux en iustallant dans le haut des vallons (les conches) des élangs artificiels. On procédait par échusées ; le flot lâché, les moulins intéressés tournaient quelques heures, s'arrêtaient ensuite. Après la disparition des moulins, les industriels de Montluçon avaient aménage une nappe d'eau naturelle du bassin, l'blang des Lancles, pour conserver dans le Cher un étiage qui ne lút pas trop délaillant. Or, c’est précisément daus cette région, sur le Cher, et non loin de là, sur la Sioule, que les premières lenlatives de barrages-réservoirs ont été menées à bien.

C'est sur la Sioule que fut donné l'exemple. On cherchait du courant pour Clemont; impossible de le demander à l'Allier, vagabond et indiscipliné. I a Sioule au contraire, au Nord-Ouest de la ville, conle à partir de Pontgibaud dans une gorge profonde qui devient encore plus étroite, plus raide et plus réserte en aval du confluent du Sioulet, lorsque la rivière passe des gneiss dans le granite. On était suffisamment rapproché de Clemonl $(40 \mathrm{~km}$.) pour transporter le courant par une ligne aérieme, même à ce début du siècle où la technique de ce transport étail encore timide. Dans la gorge, sous le village de Queuille, on fonda un barrage de 32 mètres de haut, long de 120 mètres à la partie supérieure. La vallée barrée devint un lac sinueux de 7.500 mitres de longueur, constituant une retenue de 4 millions de mètres cubes. Sous la digue, une usine fut installée, recevant sous 24 mètres de chute nette un volugne moyen de 15 mètres cubes, et produisant une puissance moyenne de $3.750 \mathrm{ki}$ - des où il serpente sur $8 \mathrm{~km}$. 500, at dams le haul cher sur $7 \mathrm{~km}$. 500. Vingt-cinq millions de mètres cubes sont emprisonnés derrière le barrage et ne sont rendus à la liberté qu'après avoir engendré 5.500 kilowatts de puissance normale dans les lurbines de l'usine, installét un peu en aval du barrage (p.l I). Désormais on n'est plus sous la hantise des lantaisies d'une rivière susceptible de tomber à 21 litres par seconde. La régulárité du débit n'est pas parfaite, loin de là ; clu moins est-on assuré désormais d'au moins 3 mètres cubes par seconde. Ià encore, le progrès clî au barrage était décisif et presque merveilleux.

Après le Cher (1909) et la Sioule (1904), ce fut le lour de l'Agout (1912). l.e bassin est plus arrosé que ceux des rivières du Nord-Est, et le débit moyen de la rivière plus considérable; la pente est forte dans la traversée du massif granitique de Vabre. La Société des Forces motrices de l'Agout se contenta d'un barrage de 17 mètres, suivi d'une dérivation de 3 kilomètres où elle fit passer un débit moyen de 9 mètres cubes, précipité sur l'usine de I uzières d'une hauteur de 117 mètres; la puissance movenne fut de 3.500 kilowatts. Ainsi, à elles seules, ces trois usines, ańénagées d'après la technique des barrages, donnaient plus de la moitié des kilowalts fournis par lés installations précédentes.

Il élait désormais acquis que seul le procédé des barragesréservoirs était capable de faire dónmer leur plein rendement 
aux forces hydrauliques du Massif Central. Les temps étaient venus de ces lacs étrangers, longs et tortueux, laissant dormir derrière un barrage leurs eaux couleur de rouille. Ces nappes d'eau, à la surface si paisible, inspirent pourtant on ne sait quelle inquietude lorsqu'on voit sortir de leur surface immobile des troncs d'arbres morts et des tètes de rocs qui semblent étonnées d'ètre submergées. De plus haut, lorsqu'on voit sur les pentes raides le manteau boisé qui les revèt descendre en hàte jusque dans l'onde et s'y engouffrer, on sent meux encore l'étrangeté de ces vallées noyées que n'anime plus le bruit des eaux.

\section{III. - RÉALISATIONS ET PROIETS ACTUELS.}

Des circonstances nouvelles allaient précipiter le mouvement, faire créer des usines nouvelles et éclore des projets. La guerre a été dans toute la France l'occasion d'une foule de réalisations hydro-électriques, mais nulle part peut-être autant que dans le Massif Central. La pénurie d'énergie y était d'autant plus grave que l'industrie s'y faisait plus active pour contribuer à la défense nationale; d'autre part, l'insuffisance éclatait de ce qui avait été réalisé jusque-là. Enfín, on avait confiance dans la technique nouvelle des barrages, qu'on jugeait capables de donner des kilowatts par centaines de mille. Ce fut un bel élan. On se rua sur les projets déjà étudiés, pour les réaliser au plus vite ; on en mit de nouveaux à l'étude, dont on entreprit en hâte la réalisation, trop en hàte parfois. Il nous faut ainsi distinguer entre les entreprises terminées, qui ne sont pour la plupart que la mise sur pied de modestes conceptions d'avantguerre, et celles qui sont en construction ou à l'étude ; de cellesci, on peut dire qu'elles font un ensemble véritablement grandiose.

I.es aménagements terminés, et en service à l'heure qu'il est, comportent un curieux mélange. Il en est qui sont conçus d'après les anciennes formules, là oì il y avait éncore des occasions à saisir. Peut-être, sans les formidables besoins de la période de guerre, ne les eût-on pas exécutés. Les autres sont des applications, soigneusement étudiées, de la technique des barrages.

Le type des premiers est l'équipement de la chute de Bar, sur la Corrèze, en amont de Tulle, et pour les besoins de la Manufacture d'armes de cette ville. On sait qu'à partir du bourg de Corrèze, la rivière s'encaisse 'et accélère sa pente jusqu'à Tulle, descendant en 16 kilomètres de 200 mètres. On a voulu profiter de cette dénivellation, bien que le débit d'étiage soit inférieur à un mètre cube. Un petit barrage de 6 mètres, retenant 36.000 mètres cubes, régularise le débit de la dérivation ; celle-ci, longue de 7 kilomètres, amène les eaux jusqu'au hameau de la Cour, où une conduite forcée utilise la chute nette de 160 mètres. La puissance moyenne est de 3.750 kilowatts ; elle tombe à 800 kilowatts à l'étiage. Les mécomptes ont été grands pendant la construction : des éboulements ont obstrué le canal. Ce retour offensif de la technique alpine n'est pas très encourageant.

La même conception est celle de la Société de Sorgue et Tarn qui, presque immédiatement avant la guerre, avait mis en service son usine du Truel sur le Tarn, létournant au moyen d'un barrage peu élevé une partie du débit de la rivière, évaluée à 26 mètres cubes au maximum, amenée au bout d'une dérivation de 1.524 mètres sur une chute de $18 \mathrm{~m}$. 50 et produisant une force moyenne de 2.500 kilowatts. Les autres sont beaucoup plus restreintes ; c'est la petite chute de Confolent sur la Creuse supérieure, en amont du bassin d'Ahun, et donnant 600 kilowatts ; relle du Palais, un peu en amont de Limoges, où une chute de 2 m. 50 en fournit 700 .

Les installations achevées depuis la guerre el comportant de grands barrages-réservoirs sont d'une lout autre importance. Tout d'abord, la Compagnie hydro-électrique d'Auvergne s'est avisée d'exécuter à plusieurs exemplaires sur la Sioule l'aménagement qui lui avait si bien réussi à Queuille. En 1917, elle mettait en service l'usine des Fades, un peu en amont du célèbre viaduc de ce nom, avec un barrage de 35 metres, au pied duquel une usine reçoit un débit moyen de 15 mètres cubes sous 25 mètres de chute netle el fournit une puissance de 3.750 kilowatts ; le réservoir gonfle derrière le lac retient un volume de 3 millions de mètres cubes (pl. II). Entre ses deux usines, dle en a installé en 1919 une troisième, celle des Garachons, comportant une retenue dont le remous remonte jusqu'au canal de fuite de l'usine des Fades, landis que l'usine nouvelle est insLallée là où vient mourir le lac de Queuille; un peu serrée entre les précédentes, celte installation est moins ample : barrage de 10 mètres, lac de 800.000 mètres cubes, hauteur de chute, grâce a une petite dérivation coupant un isthme. de $13 \mathrm{~m}$. 50 , puissance normale de 1.250 kilowatts. Cet aménagement de la Sioule sur une vingtaine de kilometres en aval du confluent du Sioulel, avec ses trois lacs étagés, ses 9.000 kilowatts de puissance normale, est un des plus remarquables qui existent dans le Massif Cientral.

D'antres élargissenuents de domaine ont été effectués. Sur l'Agout, l'usine de Luzières a reçu en 1918, par un relèvement du barrage, le renfort d'un débit supplémentaire de 16 mètres cubes : cela fait 25 mètres cubes à précipiter d'une hauteur de 117. mètres sur les turbines ; aussi l'usine peut-elle donner un maximum de 28.000 kilowatts et en puissance normale 10.500 ; elle est parmi les plus puissantes du Massif. Cependant, on en installait de plus considérables encore. Sur la Creuse, dans la zone où elle va sortir du Massif Central, on a presque terminé l'aménagement de la chute d'Eguzon. Dans ces gorges pittoresques et profondes, en amont du sile célèbre oì le ruisseau de Gargilesse, cher à George Sand, dégringole vers la Creuse, on a édifié au travers du lit une muraille de 60 mètres, en arrière de laquelle le remous s'étendra sur 16 kilomètres de longueur, de méandre en méandre, sous Crozant qui le domine du haut de son promontoire, et jusque dans la Pelite-Creuse ; le débit moven des conduites forcées sera de 22 mètres cubes et la puissance normale de 15.000 kilowatts. D'ici longtemps probablement elle restera le plus gros organisme du Massif ; elle est destinée à l'électrification de la section Châteauroux-Paris du chemin de fer P.-O. et, en attendant, sera en liaison avec l'usine thermique de Gennevilliers pour l'aider à passer ses pointes. La Vienne n'a pas voulu être en reste. Encore encaissée dans les granites à 90 mètres au-dessous du plateau poitevin, elle présente, entre Confolens et l'Isle--Jourdain, une vallée assez étroite pour être utilement barrée. C'est ce qui a été fait immédiatement en amont de l'Isle-Jourdain : barrage de 11 mètres, avec lac remontant à 4,5 kilomètres ; débit moyen de 45 mètres cubes, et possibilités d'utilisation jusqu'à 60 ; puissance normale de 3.000 kilowatț, employée à desservir toute la région qui va de Châtelleraull à Saintes par Poitiers, Niort, La Rochelle. Autres usines nouvelles : celle du Viaur à Thuriez, vers les limites du Tarn et de l'Aveyron ; barrage de 30 mètres, assurant, avec une petile dérivation, une chute de 31 mètres ; réservoir de 5 kilomètres de long ; débit moyen de 11,75 mètres cubes ; puissance normale de 2.330 kilowatts, envoyée par une ligne de 40 kilomètres aux usines de zinc de la Vieille-Montagne, à Viviez; enfin la seule de ces installations qui soit au centre mème du Massif, celle du Moulin-de-Vergne sur le Bès, affluent de la Truyère. Dans celleci, nous trouvons déjà les traits caractéristiques que nous reverrons sur les projets de la Cère et de la Truyère : barrage-réservoir et dérivation combinés. Deux barrages. un sur le Bìs, ull 
sur son affluent la Bédaule, dont les eaux sont déversées dans le réservoir principal ; une dérivation en tunnel, dans le granite, de 4,500 mères, une chute brute de 144 metres, un débil de 10 mètres cubes, une puissance normale de 6.500 kilowatts, envovée sur 15 kilomètres de fils aux usines électro-mélallurgiques que la Compagnie des Aciéries el Forges de Firminy a installées à Saint-Chély-d'Apcher, en pleine Lozère.

'Tels sont les résultats déjà définitifs que l'effort de guerre a engendrés ici. On voit qu'ils ne sont pas négligeables. En laissant de côté le Truel, terminé avant la guerre, et en y joignant Eguzon, qui n'est pas encore en service, ils représentent une puissance normale de 44.000 kilowatts, c'est-à-dire qu'ils ont plus que doublé les 36.000 kilowatis en service en 1914. Ils consacrent le succès de la méthode des barrages, et celui-ci est si net qu'aux abords des barrages déjà édifiés, les projets el les études fourmillent. A l'Isle-Jourdain, il est question de créer deux nouvelles retenues, une en amont, avec barrage de $8 \mathrm{~m} .50$, l'autre en aval, haute de 7 mètres ; on disposerait ainsi de 4.500 nouveaux kilowatts. Sur le haut Cher, les études sont très avancées à Lignerolles, au-dessous de l'usine de Rochebut ; un barrage de 42 mètres créerait un remous de 6.500 mètres de long, venant expirer au canal de fuite de Rochebut; la chute, de 38 mètres, donnerait 4 à 5.000 kilowatts. Le total des eaux emprisonnées dans les deux réservoirs approcherait de 40 millions de mètres cubes et permettrait de garder l'été 5 à 6 mètres cubes le débit, landis qu'on tombait autrefois au-dessous de 500 litres. Il $\mathrm{y}$ a des projets pour l'Isle supérieure et de grands barrages prévus sur la Vézère et la Corrèze. Remarquons toutefois que lout cela, sauf le Bès, est périphérique, et par suite ne s'attaque pas anx grosses réserves de force du Massif, les rivières du centre, les cours d'eau des hautes terres. C'est bien naturel ; on est allé d'abord au plus proche, aux emplacements les plus voisins des plaines privées de combustible et réclamant de l'énergie. Mais du moment qu'on voulait faire grand, c'est à l'intérieur qu'il fallait chercher. C'est donc à l'intérieur du Massif que sont les projets récents et les débuts d'exécution ; c'est là aussi que sont les amples conceptions, les audacieuses techniques.

Deux parts sont à faire dans ces projets et réalisations actuels : ceux qui concernent des bassins déjà attaqués, Loire, Allier, Vieune, Tarn ; ceux qui s'attaquent aux puissantes rivières presque vierges du Centre proprement dit, haute Dordogne et affluents, Cère, Truyère.

Le tour des premiers est vite fait. On l'a vu, le bassin de la Loire est déjà fortement écrémé. Une usine projetée sur le Lignon forézien (Saint-Georges-en-Couzan) domnerait 1.400 kilowatts ; deux autres sur la Loire supérieure (Issarlès, Coubon), en fourniraient ensemble 2.600. Mais l'utilisation du lac d'Issarlès comme réservoir saisonnier pourrait tripler ce rendement. La ville de Saint-Ftienne enfin a étudiè sur le Lignon vellave l'exhaussement, jusqu'à 54 mètres de haut, du barrage de Lavalette qu'elle a fait établir pour se constituer une réserve en eau potable; une trentaine de millions de mètres cubes seraient ainsi emmagasinés, et leur emploi en deux chutes pourrait donner 9 à 10.000 kilowatts. Le haut Allier, capricieux, difficile à aménager, commence cependant à être tâté dans l'étroit défilé à l'amont de Langeac où il est serré entre la Margeride et les coulées du Velay. Deux barrages ont été disposés sur l'Allier et son affluent l'Ance, et deux dérivations, l'une de 3.153 mètres, l'autre de 4,5 kilomètres, doivent amener à l'usine de Monistrol un volume évalué, peut-être trop largement, à 20 mètres cubes pour l'Allier, à 10 pour l'Ance ; les chutes sont de 50 mètres pour l'eau de l'Allier, de 199 pour celle de son aff́luent, et la puissance normale qu'on espère en tirer est évaluée à 8.500 kilowatts. Sur le-Tarn, l'aménagement en cours se ramène à l'utilisation d'un de ces cas exceptionnels par lesquels a débuté la mise en valeur des cours d'eau du Massif : en recoupant le pédoncule, étroit de 25 mètres, qui sépare les deux extrémités du fameux méandre d'Ambialet, long de 3 kilomètres, on obtient une chute de $4 \mathrm{~m}$. 50 et une puissance de 1.950 kilowatts destinée aux usines proches du Saut-du-Tarn. Enfin sur le Taurion, la Société hydro-électrique du Palais demande la concession de deux chutes fournissant 9.000 kilowatts, et le projet semble près d'aboutir. L'aménagement doit porter sur le bief en pente raide à l'avai de Bourganeuf ; à la Roche-Talamie, un barrage de 25 mètres, soutenant un lac de 9 kilomètres, étendu sur 200 hectares; de là partirait une dérivation de 6,3 kilomètres, donnant à la Salesse une chute de 97 mítres, avec un débit de 9 à 20 mètres cubes ; en aval, à Saint-Martin, un deuxième barrage (34 mètres), un lac de 10 kilomètres, une chute par dérivation de $34 \mathrm{~m} .50$. C'est là un projet plein d'intérêt, qui aiderait fort à régulariser le débit de la Vienne et pourrait dès lors entraîner d'autres aménagements sur cette rivière.

Mais nous avons hâte d'en venir aux grands programmes du centre, ceux qui expriment le mieux les possibilités hydro-électriques du Massif Central. Ici encore une distinction s'impose, fondée sur des considérations d'ordre plyxsique, entre Truyère, Cère et Rhue d'une part, Dordogne de l'autre.

La Cére, la Truyère, rivières puissantes, sont pourtant assez différentes l'une et l'autre de la Dordogne, au moins en ce qui concerne le profil de leur cours et les formes de leurs vallées. Le hasard semble en ètre entièrement responsable. Les deux rivières ont l'occasion de traverser, au cours de leur carrière, des bassins de dépôts tertiaires qui trouent la plate-forme rigide du Massif Central. Ces dépôts (sables et argiles) sont tendres ; la rivière y a aisément enfoncé son lit, élargi ses rives : sa pente s'y atténue. Ainsi la Cère, au Sud du bassin d'Aurillac, entre Arpajon et le pont du Bex, n'a pas 2 mètres de pente par kilomètre. Au contraire, à l'amont et même à l'aval, en pleines roches dures, gneiss, puis granite et surtout granulite, la déclirité redevient considérable ; sur la Cère, $4 \mathrm{~m}$. 60 au kilomètre entre le pont du Bex et Laroquebrou, et près de 12 mètres au kilomètre entre Laroquebrou et Laval-de-Cère, où la rivière pénètre dans le kassin de Beaulieu. Sur la Truyère, déclivité insignifiante, réduite à moins d'un mètre par kilomètre, dans le bassin du Malzieu ; 6 à 7 mètres ensuite, dans la section qui va jusqu'au viaduc de Garabit ; $1 \mathrm{~m}$. 70 entre Garabit et le pont de Lanau, sur l'emplacement d'un ancien bassin tertiaire presque entièrement nettoyé de ses dépôts, et peut-être aussi sous l'influence du barrage que formaient à l'aval les basaltes du Cantal rejoignant ceux de l'Aubrac. En tout cas, au delà, dégringolade : $8 \mathrm{~m}$. 80 au kilomètre entre le confluent du Brezons et le pont d'Entraygues, dont 25 environ par kilomètre en aval du pont de la Cadène, sur une distance de 9 à 10.000 mètres.

Cette irrégularité du profil permet une appropriation spéciale. En construisant un barrage à l'entrée aval d'une zone de faible pente, on peut, avec une médiocre hauteur d'obstacle, submerger une vaste étendue et constituer ainsi à peu de frais un ample réservoir régulateur. Ceci fait, en accrochant aux parois de la gorge qui s'enfonce vers l'aval une derivation partant du niveau du barrage, on obtient très aisément, en quelques kilomètres, une haute chute dont le debit est régularisé par le réservoir d'amont. Ainsi la Truyère, la Cère, leurs affluents, doivent ètre le domaine d'aménagements combinant les barrages et les dérivations, la méthode des Alpes et celle du Massif Central, pour obtenir des résultals remarquables. Ces aménagements sont déjà commencés.

Des deux rivières, la Cère est probablement la moins irrégu- 
lière et son débit moyen, 26 mètres cubes à taroquebrou, est déjà intéressant. Elle a aussi lavantage de dérouler son cours dans une contrée accessible, desservie par plusieurs voies ferrées, dont lume, renant de la loordogne, remonte toute la vallée pour gagner Aurillac et le Lioran. Ces considérations ont probablement décidé du choix des Aciéries et Forges de Firminy, qui, en 1917, ont commencé l'aménagement du cours inférieur de la rivière. Un petit barrage de 10 mètres est édifié à 3 kilomètres en aval de Laroquebrou : de là part une formidable dérivation de vingt kilomètres, débouchant sur Laval-de-Cère et aboutissant à une chute brute de 260 mètres. On saisit, à voir quels chiffres donne ici l'emploi du procedé alpin de la dérivation, quelle différence sépare le Massif Central des Alpes, quant aux iptitudes hydro-hélecLriques ! La dérivation est prévue pour débiter 20 mètres cubes à la seconde : on compte qu'elle en dommera en moyenne 14.6. Six groupes de 8.000 chevaux chacun donneront une puissance maxima de $+\$ .000$ chevaux, une puis- sance nomale de 25.000 kilowats. Trois kilomètres de la dérivation sont déjà conslruits à l'amonl ; on aménage à leur issue une petite usine de 1.200 kilowatts qui four nira la force aux perforatrices et en même. temps éclairera Aurillac.

Le poinl faible de cette grandiose entreprise, qui dépassera

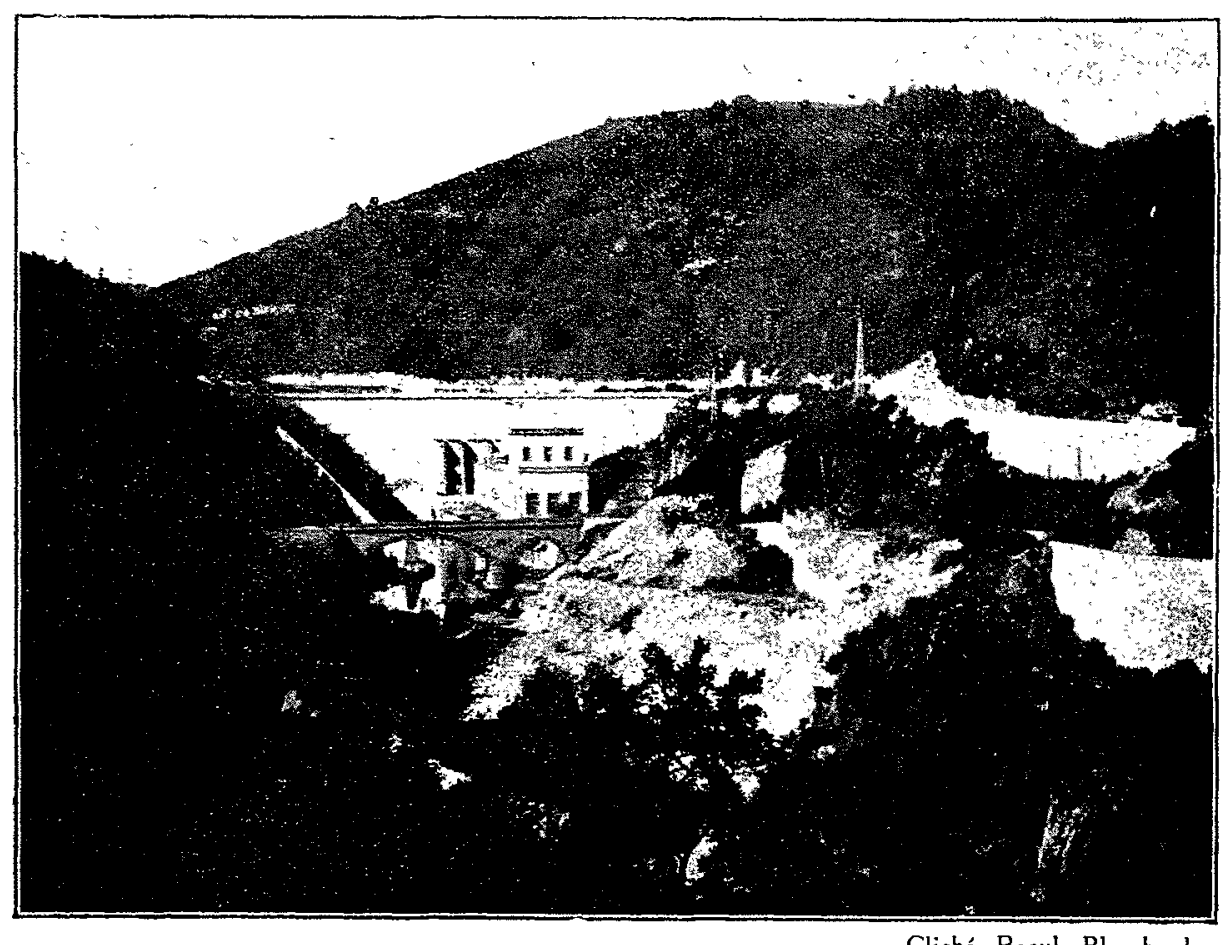

Pl. II-A. - Barrage et usine des Fades (Sioule).
Mais la perle de la Truvère, c'est comme pour la Cère l'aménagement du bief inféricur. Il est vrai qu'ici, l'ètablissement, de réservoirs régulateurs est plus nécessaire encore qu'ailleurs, le débit pouvant varier au pont de la Cadène de 2 à 1.200 mètres cubes. Aussi deux barrages au moins sont-ils próvus dans la section en pente douce qui va de Garabit à la Cadène. A l'amont, celui du Pradal, sous le confluent du Bès, aurait 65 mètres de hauteur et emprisonnerait 200 millions de mètres cubes. Plus bas, celui de Sarrans. à 1.500 mètres du pont de la Cadène, s'élèverait à 55 mètres, avec 100 millions de mètres cubes derriere lui. Dans ces conditions, le débit du torrent serait régularisé à 40 mètres cubes et de magnifiques perspectives s'ouvriraient pour l'usine du cours inférieur. Celle-ci, dont l'aménagement est commencé depuis 1914, par un groupe puissant où sont intéressées des sociétés houillères, comporte un petil barrage de 14 metres, une dérivation de $7.2633^{\circ}$ mètres en plein granite : la chule brute sur lusine de Valcayles est de 2,ji mèlres. A 15 malres cubes de débil caracleristique moyen. (cest une puissance nomale de 30.000 kilowalls: a 40 mètres cubes, ce serail 80.000 , el lensemble, qu'on désignt ordinairement sous le nom de la commune interessé, Brommal, serail de beaucoulp, apros Cienissial, lo plus grandiose the France. en puissance les plus fortes usines alpines, c'est la trop faible capacité du réservoir entre le barrage et Laroquebrou. La Cère a un débit caractéristique d'étiage de 2,6 mètres cubes ; à la saison d'étiage, au lieu de 20 mètres cubes, c'est donc 2 ou 3 qui seront dérivés, et l'on aura 4.000 kilowatts au maximum. Il faut donc régulariser la Cère, et, puisque la présence de la bourgade de Laroquebrou empèche de relever le barrage de la grande chule, il laut en construire un autre en amont, vers SaintEtienne-de-Cantales, susceptible d'étoffer un peu le flot de la Cère pendant l'été. Tant il est rrai qu'une simple dérivation ne suffit pas ici à assurer un rendement profitable, ni une usine qui paye.

Aussi les aménagements commencés ou projetés sur la Truyère comportent-ils des barrages assurant le service des dérivations. A un kilomètre en aval du Malzieu, au pied de la Margeride, un petit barrage de 3 mètres de laut est en construction ; conjugué à une dérivation, il assurera à la Société de Firminy une puissance normale de 1.250 kilowatts, qu'on espère soutenir en réalisant à l'amont (Prunières) de petits réservoirs saisonniers. Un peu au delà, un nouveau barrage de 20 mètres déterminerait un remous de 2 kilomètres ; une dérivation 's'ensuivrait sur 3 kilomètres, donnant une chute d'une soixantaine de mètres et une puissance normale de 3.000 kilowalts. Avec l'usine du Bès, la Socielé de Firminy se constitue done jei une foree considerable ponr alimenter son usine de Sainl-Choly-d' $\Lambda$ peher.
Reste la Rhue, affluent qui apporte à la Dordogne, tout pris de Bort, les eaux de presque toutes les pentes septentrionales du Cantal, du Cézallier oriental, et mème du Sud du Sancy. Ces illustres parentés lui valent une nature spéciale; la Rhue est a coup sûr, de ses sources à son embouchure, le lype le moins imparfait de cours d'eau de montagne qu'il y ait dans le Massil Central. Chacune des branches grossièrement convergentes qui la constituent descend de sommets élevés, Puy Mary (1787), Puy de Sancy (1886), croupes du Cézallier (1478); leur pente est done rapide, el leur alimentation abondanle. Quant au cours infériem, il présente une déclivilé fort irrégulière, où des paliers alternent avec des cascades comme le Saut-de-la-Saule. Tout celi serait parfait pour l'industrie hydro-électrique si le débit avait de la régularité. Mais, bien que la neige puisse persister dans le haut du bassin jusqu'en fin mai, l'étiage r'août-octobre n'en est pas moins très dur à traverser, et le débit parâ̂t tomber all conlhent avec la Dordogne à 2 mètres cubes à la seconde. Li aussi, il faut donc des barrages pour constiluer des réserves ati somnières, el en aval des dérivations susceptibles de protiler de tous les caprices de la pente.

L'aménagement comporte donc d'abord la création de réser. voirs, dans les parlies favorables du cours supérieur des diver aflluents en éventail. Le lac Chauvet aura son niveau relevé jusplu'a rontenir 10 millions de metres cubes ; la Phue d'Eglist neuve, barróa alu-dessous du village de Chanterelle, domera th: 
rescrvorr de 11,7 millions de mètres cubes ; sur la Petite-Rhue, la digue de la Font-Salée en retiendra 27 millions: Voilà donc 50) millions d'emmagasinés, de quoi alimenter les conduites. Un lumnel de 7.500 mètres de long amènera un débit maximum de 12 mètres cubes pris à la Grande-Rhue et déversera ces eaux d'une hauteur de 153 mètres sur une usine installée au confluent avec la Petite-Rhue ; le long de celle-ci, un tumel de 9 kilomètres guidera au même rendez-vous, pour une chute de 245 mètres, un débit de 6 mètres cubes. L'usine du confluent aura ainsi une puissance moyenne de 12.000 kilowatts. Au-dessous, les eaux régularisées sont détournées dans un tunnel de $4 . \overline{5} 00$ mètres ; à l'extrémité, l'usine de Sarran, $47 \mathrm{~m}$. 50 de chute, 20 mètres cubes de débil maximum, 3.500 kilowalts de puissance. Cn dernier barrage (Rocliemont), dominant le Saut-de-la-Saule, noiera le palier d'amont sous 29 millions de mètres cubes; et voilà pour l'usine à construire un peu cn aval un débit maxinum de (1) mètres cubes à faire lomber de 62 mètres de hauteur; ci, encore 6.850 kilowatts. Total pour la Rhue, une puissance normale supérieure $\grave{a} 22.000$ kilowatts, que la Compagnie d'Orléans compte appliquer à l'électrification de son réseau; elle en a confié l'aménagement aux usines Schneider, et les travaux de la chule la plus élevée ont dû commencer à la fin de lautomne dernier.

La Dordogne. - Maintenant, voici le plus fort! Avec la haute Dordogne el les projets qui la concernent, nous atteignons des chiffr̆es colossaux, par une application grandiose de la méthode des barrages. C'est que la rivière présente des caractères particuliers. Sans doute, elle naît au pied du Sancy, mais ne s'attarde guère en haute montagne ; vingt kilomètres après sa source, elle est déjà tombée au-dessous de 750 mètres. D'autre part, aucun bassin de dépôts tertiaires ne s'interpose sur le parcours de la rivière avant qu'elle ait quitté le Massif Central à Beaulieu. La seule roche tendre qu'elle rencontre sur son passage, ce sont les grès et schistes houillers qui ont valu à la vallée la dilatation du bassin de Bort et un adoucissement local de la pente. Partout ailleurs, à travers les gneiss, la granulite, le granite, le talweg déroule une pente régulière, qui va s'accentuant peu à peu vers l'amont. Sauf au droit de la rupture de pente du bassin de Bort, il n'y a donc pas lieu ici d'installer des dérivations; la technique d'aménagement sera, dans son plein épanouissement, celle des barrages-réservoirs.

Les premières installations, réservées comme celles de la Rhue à la Compagnie d'Orléans, se feront non sur la Dordogne, mais sur son affluent supérieur, le Chavanon, plus long qu'elle, et dont la vallée, profonde et absolument déserte, se prête mieux aux submersions que celle de la Dordogne. Le premier barrage, dont les travaux ont dû récemment être adjugés, sera fondé près du hameau de la Cellette, sous Bourg-Lastic, au droit d'une gorge dont la largeur à la base ne clépasse pas 30 mètres, entre des parois d'excellent gneiss. Avec lui, nous entrons dans la série des barrages géants : 81 mètres de hauteur, el derrièrè dáns la vallée profonde dont la déclivité ne se relève qu'ave. lenteur, un réservoir de 190 millions de mètres cubes, long de 15 kilomètres et ramifié dans une foule de vallées affluentes. Pour mieux alimenter ce réservoir, on y dérivera par un tunnel de 6 kilomètres de longueur la haute Dordogne et une autre rivière, la Clidane. L'usine, sous la Cellette, aura une chute brute supérieure à 80 mètres et réalisera une puissance moyenne de 4.800 kilowatts. Ce n'est pas énorme; mais le grand intérêt de cette installation, c'est de commencer la régularisalion du débit et d'exercer ainsi une action bienfaisante qui se répercutera sur le fonctionnement do toutes les usines d'aval.

Au delà, sous l'influence du bassin de Bort, la vallée Chavanon-Dordogne tend à s'élargir; une voie ferrée s'y installe, celle d'Eygurande à Bort; une submersion par barrage n'est plus possible. Il faut done recourir aux dérivations. Lne première prendra l'eau au canal de fuite de la Cellette et, par un tunnel de 10 kilomètres sous la rive gauche, aboutira droit au confluent Dordogne-Chavanon où une usine utilisera une chute brute de 97 mètres, un débit maximum de 18 mètres cubes et fournira 3.200 kilowatts. Deux kilomètres plus bas, nouvelle dérivation en tunnel, 15 kilomètres et demi, la plus longue après celle de la Cère; elle happera áu passage quelques ruisseaux et amènera un peu en amont de Bort un débit maximum de 24 mètres cubes, dégringolant de 103 mètres de chute brute el engendrant 9.350 kilowatts. Voilà, pour la Dordogne supérieure, une puissance moyenne de près de 20.000 illowatts que la Compagnie d'Orléans veut employer à l'électrification de son réseau.

Au delà de Bort, un peu en aval du confluent de la Rhue, la Dordogne réintègre une gorge qui, à travers le granite, les gneiss, les micaschistes, la granulite, les micaschistes encore, ne cesse guère qu'au confluent du Doustre, trois kilomètres avant Argentat. Pour 80 kilomètres, il y a là 240 mètres de pente. C'est tout à fait insuffisant pour des dérivations ; en revanche, comme la vallée est étroite et presque déserte, les barrages-réservoirs y sont tout indiqués. Lorsque, cheminant au Sud-Ouest de Bort sur les larges plateaux ondulés, on atteint brusquement les abîmes de 200 à 300 mètres de profondeur où sont ensevelis la Dordogne et ses affluents, qu'on domine leurs pentes roides où le roc perce, grisâtre, à travers le manteau d'une maigre végétation, et qu'en se penchant sur la gorge qui tranche si net le plateau on n'entend monter du fond que le bruit du torrent mâchant ses cailloux; on se persuade aisérnent que le seul emploi 


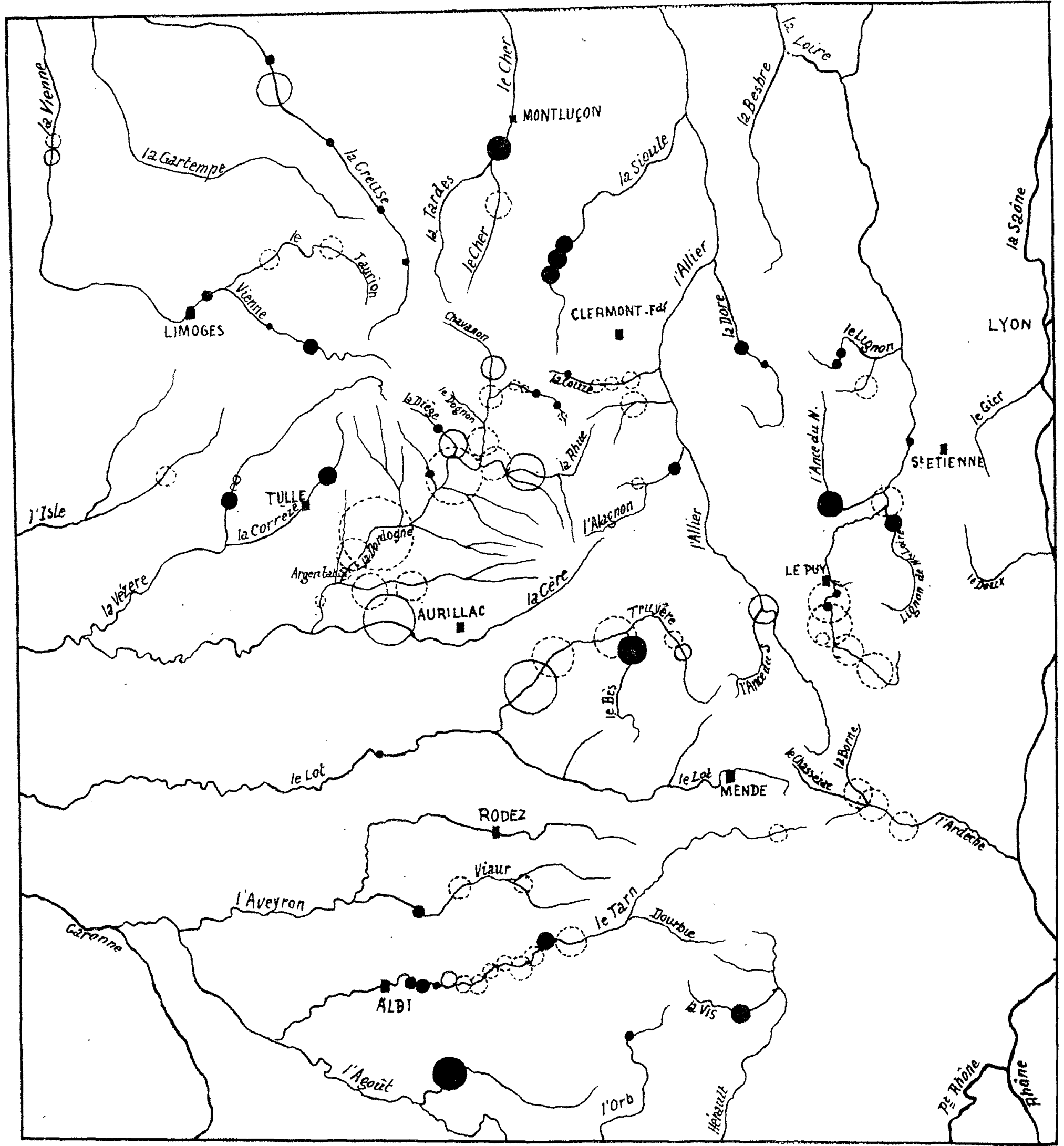

Fig. 1. - Les Usines Hydro-électriques du Massif Central.

Les surfaces noires représentent les usines en fonctionnement; les cercles à trait plein, les usines en construclion; les pointillés, les usines en projet déjà ćludic. Là grandeur des circonférences ést proportionnelle à la puissance.

"humain " de ces profondes rainures est d'être transformées en régulateurs de houille blanche (pl. II). Pas toul à fait, cependant, et il laudra sacrifier quelques ponts, quelques kilomètres de route, environ 200 hectares de propriétés el même quelques hameaux pour donner aux gorges leur nouvelle et bienfaisante destination. L'intérêt général en est tel que l'hésitation n'est guère permise.
Trois grands barrages sont done prévus entre Borl e! Argetl. lat. $\Lambda$ l'amont, le barrage du pont de Vernéjoux est prévu pour 92 metres de hauteur (1). Le lac enserré derrière, inondant li gorge Lortueuse de la Dorrlogne jusqu'au confluent de la Rhue

(1) La hauteur de ces barrages est indiquée fondations nom comprises. 
(18 kilomètres), contiendra 90 millions de metres cubes. La chute nette, supérieure à 80 mètres, donnera une puissance de 26.550 kilowatts. Tout à l'aval, le barrage de Graffeuille, à 4,6 kilomètres d'Argentat et un peu en amont du confluent du Doustre, aura 45 mètres de hauteur, retiendra une réserve de 25 millions de mètres cubes et donnera une puissance de 20.700 kilowatts, le débit de la Dordogne s'étant accru depuis Vernéjoux de l'apport de nombreux affluents. Les affluents, eux aussi, sont susceptibles d'utilisation. La Société des Hauts-Fourneaux et Fonderies de Pont-à-Mousson a commencé en 1918 d'importants travaux sur la Diège : barrage de 35 mètres en amont du moulin de Ratabourg ; réservoir de 6 kilomètres de long, faisant 9 millions de mètres cubes; un tunnel de 2.800 mètres, coupant rne boucle, permet de placer la chute sur la Dordogne même, ce qui la porte à 130 mètres et paraît devoir assurer 7.600 kilowatts moyens. Encore à 300 mètres en amont du pont d'Argentat, un barrage à vannes de $6 \mathrm{~m}$. 50 donnerait enfin, sur une rivière désormais régularisée, une puissance de 3.550 kilowatts.

Cette régularisation proviendrait non seulement de l'effet des barrages du Chavanon, de Vernéjoux et de Graffeuille, majs surtout de la pièce maîtresse du système, le barrage monstre de Chambon. Celui-ci serait placé entre Graffeuille et Vernéjoux, à 4 kilomètres en aval du pont de Chambon. La hauteur prévue est de 114 mètres, ce qui assurerait à l'usine blottie à sa base une chute brute de 109 mètres et une puissance de 55.000 kilowatts. Le lac-réservoir serait de dimensions colossales': une longueur de 40 kilomètres, sans compter les indentations aflluentes ; une contenance enfin de 525 millions de mètres cubes. Il serait le plus haut barrage du monde et emmagasinerait plus d'eau que Génissiat (Rhône), un peu moins que Serre-Ponçon (Durance). L'entreprise n'offre aucune impossibilité technique, et après l'avis d'une commission spéciale, le Conseil supérieur des Travaux publics a donné son adhésion au projet. Sans doute il coûtera cher, 30 millions au mcins, et plutòt 40 que 30 . Mais songeons qu'il fournira 50.000 à 100.000 kilowatts, et surtout qu'en portant en toute saison le débit de la Dordogne à 75 mètres cubes au moins, l'étiage actuel sera quadruplé, ce qui permettra la navigation en toute saison el une production régularisée de kilowatts aux usines existantes de Mauzac, Tuilières (non loin de Bergerac), comme aux installations à créer en aval d'Argentat au moyen de basses chutes variant de 3 à 13 mètres ; on calcule qu'il y a là possibilité de produire 95.500 kilowatts, désormais presque permanents puisque, grâce au barrage, les basses eaux seront fortement gonflées et les hautes eaux atténuées. Avec Chambon, le roi des barrages, cette technique originale du Massif Central en arrive à l'apothéose (1).

\section{$* * *$}

Faisons le compte des résultats acquis et de ceux qu'on escompte. D'abord une puissance moyenne, dès maintenant acquise, de 80.000 kilowatts. Dans les installations en voie d'aménagement, sur l'Allier, le Tarn, la Cère, la Truyère, la Rhue, le Chavanon, la Diège, se créent des disponibilités normales de $91.000 \mathrm{ki}-$ lowalls. Enfin les autres projets qui semblent déjà mûrs, et certains bien près d'aboutir, représentent encore 158.000 kilowatts,

(1) Il faut avouer que la sécheresse de 1920-1921 a abaissé le débil des rivières du Massif à des chiffres tels que les réservoirs projetés seraient encore insuffisants. Mais il nous parait que cette sécheresse est un phénomène assez extraordinaire pour qu'on puisse se dispenser de tabler sur elle. ront 106.000 pour la Dordogne enıre Bort et Argentat. Cela fait bel et bien un total de 330.000 kilowatts en puissance moyenne ; le Massif Central pourra soutenir honorablement la comparaison avec les Alpes.

N'est-ce pas même un peu trop? N'y a-t-il pas eu, depuis 5 ans, une poussée un peu trop vive? On a agi, pendant la guerre, comme si la pénurie de charbon devait être un phénomène permanent et comme s'il fallait s'arranger désormais pour remplacer la houille par l'énergie hydro-électrique, à tout prix et par tous les moyens. In certain nombre de projets, quelques aménagements en train, peuvent donc être considérés comme appartenant au type "usines de guerre ", avec tout ce que cette acception représente de hâtif, de risqué, d'artificiel même. Il est remarquable aussi que l'immense majorité des kilowatts produits sur les chutes du massif ne soient pas employés sur place, comme c'est si souvent le cas dans les Alpes, mais expédiés à distance pour engendrer force et lumière. L'électro-métallurgie, si développée dans les Alpes, n'est représentée ici que par les usines de Firminy à Saint-Chély-d'Apcher et - pour partie - par les établissements du Saut-du-Tarn. L'électrochimie n'existe pas. Il ne se rencontre pas non plus dans le Massif Central, comme dans les Alpes, de ces grosses papeteries qui ont aménagé à leur usage des forces considérables. Toute, ou à peu près toute l'énergie produite ici est à vendre, à écouler le mieux possible par des réseaux appropriés de distribution.

Et c'est ici que nous paraît être le point capital. Toute cette énergie qu'on projette de créer, dont on a commencé d'aménager les installations, il ne sera vraisemblablement pas difficile d'en assurer le placement, puisque le Massif Central est le mieux placé pour fournir de la force à presque la moitié de la France, de la Loire à la Garonne et de l'Océan au Rhône. Mais pour cela il faut créer, en même temps que les chutes, les grands réseaux de distribution, susceptibles de satisfaire à toutes les demandes et de grouper toutes les ressources. On peut égalemenrt envisager la jonction avec le réseau alpin, assez indiquée puisque les deux étiages y sont à des saisons opposées. N'oublions pas enfin que les compagnies de chemins de fer revendiquent une bonne part de ces forces. Les aménagements commencés ou projetés pour le compte du P.-O. sur le Chavanon, la Dordogne et la Rhue représentent plus de 68.000 kilowatts. Voilà donc plus du cinquième des forces créées ou projetées qui se trouve déjà placé

Il nous parait donc qu'on peut $y$ aller avec confiance èt créer ici de magnifiques ressources d'énergie. Assurément ce sont déjà, ce seront plus encore des kilowatts chers. La technique des barrages-réservoirs, celle des longues dérivations, ne peuvent s'accommoder des prix favorables de premier établissement qu'on a obtenus au début dans les Alpes. Cela n'a pas de conséquuences trop graves : ici les kilowatts sont vendus, pour lumière et force, à des gens qui peuvent subir des prix plus élevés que les électro-métallurgistes par exemple ; pour la plupart des industriels, la dépense de courant n'intervient dans le prix de la fabrication que pour une proportion variant entre 2 et $10 \%$. Songeons aussi que ces kilowatts seront de plus en plus, à mesure que s'élèveront les constructions cyclopéennes des barrages, un produit régularisé, tendant peu à peu à devenir un produit permanent, c'est-à-dire qu'ils seront à peu près affranchis du gros défaut qui est le péché de la houille blanche, l'irrégularité. Partie dans des conditions manifestes d'infériorité qu'elle devait à la nature physique de ses montagnes, la houille blanche du Massif Central risque fort, pour y avoir laborieusement remédié, de prendre le pas sur les autres utilisations hydro-électriques francauses. 